\title{
Case Report \\ Regressing Multiple Viral Plaques and Skin Fragility Syndrome in a Cat Coinfected with FcaPV2 and FcaPV3
}

\author{
Alberto Alberti, ${ }^{1}$ Gessica Tore, ${ }^{1}$ Alessandra Scagliarini, ${ }^{2}$ Laura Gallina, ${ }^{2}$ \\ Federica Savini, ${ }^{2}$ Chiara Caporali, ${ }^{3}$ and Francesca Abramo ${ }^{4}$ \\ ${ }^{1}$ Department of Veterinary Medicine, University of Sassari, Via Vienna 2, 07100 Sassari, Italy \\ ${ }^{2}$ Department of Veterinary Medical Sciences, University of Bologna, Via Zamboni 33, 40126 Bologna, Italy \\ ${ }^{3}$ Private Practitioner, Via Giovanni da Verrazzano 19, 52100 Arezzo, Italy \\ ${ }^{4}$ Department of Veterinary Sciences, University of Pisa, Viale delle Piagge 2, 56124 Pisa, Italy
}

Correspondence should be addressed to Francesca Abramo; francesca.abramo@unipi.it

Received 28 September 2015; Accepted 1 December 2015

Academic Editor: Katerina K. Adamama-Moraitou

Copyright (C) 2015 Alberto Alberti et al. This is an open access article distributed under the Creative Commons Attribution License, which permits unrestricted use, distribution, and reproduction in any medium, provided the original work is properly cited.

\begin{abstract}
Feline viral plaques are uncommon skin lesions clinically characterized by multiple, often pigmented, and slightly raised lesions. Numerous reports suggest that papillomaviruses (PVs) are involved in their development. Immunosuppressed and immunocompetent cats are both affected, the biological behavior is variable, and the regression is possible but rarely documented. Here we report a case of a FIV-positive cat with skin fragility syndrome and regressing multiple viral plaques in which the contemporary presence of two PV types (FcaPV2 and FcaPV3) was demonstrated by combining a quantitative molecular approach to histopathology. The cat, under glucocorticoid therapy for stomatitis and pruritus, developed skin fragility and numerous grouped slightly raised nonulcerated pigmented macules and plaques with histological features of epidermal thickness, mild dysplasia, and presence of koilocytes. Absolute quantification of the viral DNA copies (4555 copies/microliter of FcaPV 2 and 8655 copies/microliter of FcaPV3) was obtained. Eighteen months after discontinuation of glucocorticoid therapy skin fragility and viral plaques had resolved. The role of the two viruses cannot be established and it remains undetermined how each of the viruses has contributed to the onset of VP; the spontaneous remission of skin lesions might have been induced by FIV status change over time due to glucocorticoid withdraw and by glucocorticoids withdraw itself.
\end{abstract}

\section{Introduction}

Feline viral plaques (VPs) are well recognized uncommon skin lesions clinically appearing as solitary or multiple, raised, often pigmented, small plaques [1-4]. Cats are affected at any age [4] and they may be either immunocompromised $[1,2,5]$ or immunocompetent [4]. Microscopically, a VP consists of well-demarcated foci of epidermal thickening, mild dysplasia, and presence of koilocytes. VPs are associated with the presence of papillomavirus (PV), predominantly with Felis catus papillomavirus type 2 (FcaPV2), but other PV types as well as multiple PVs in a single lesion have been less frequently reported [4-6]. The behavior of VPs is variable; progression to Bowenoid in situ carcinoma (BISC) and invasive squamous cell carcinoma (SCC) is a risk; the plaques can remain small for long time and regress; however, very little information about follow-up is available from the published cases [7]. This paper reports a coinfection with 2 PV types, FcaPV2 and FcaPV3, in a FIV-positive cat with skin fragility syndrome (SFS) and spontaneous regressing multiple VPs. Papillomaviruses were molecularly characterized and relative viral loads were quantified.

\section{Case Presentation}

A short-haired adult neutered male cat was presented to a private veterinary clinic for bilateral pruritic alopecia and skin lesions on the trunk, noticed by the owners 5 months before, when the cat was found as a stray. The cat had been treated for a severe stomatitis and severe pruritus with two $20 \mathrm{mg} / \mathrm{cat}$ subcutaneous injections of 


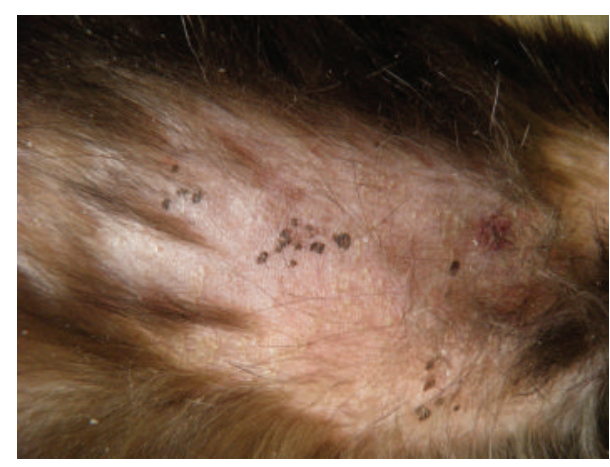

(a)

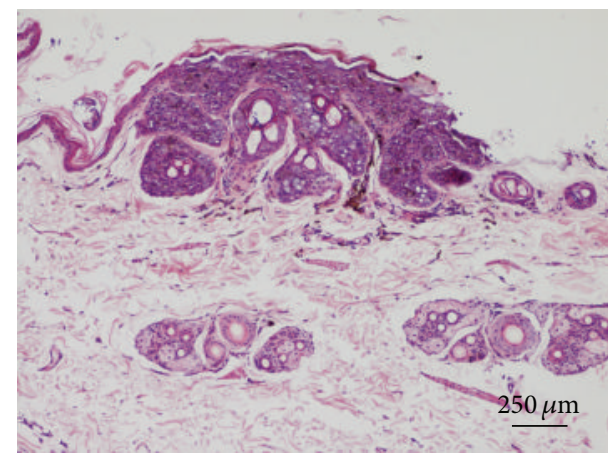

(c)

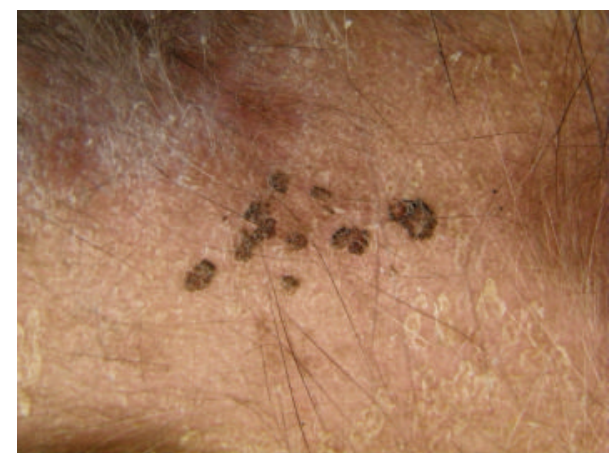

(b)

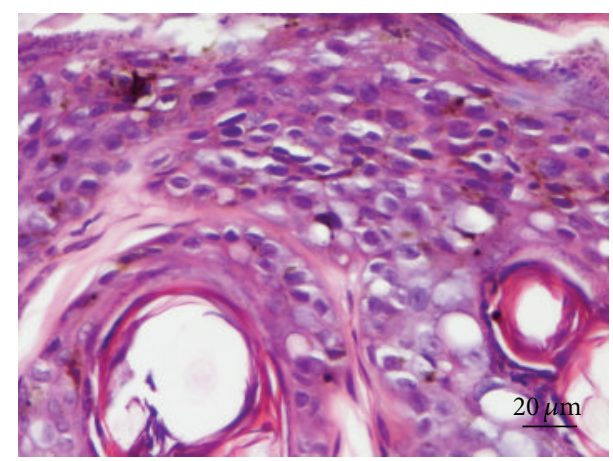

(d)

FIGURE 1: Macroscopic and microscopic features. Ill-defined alopecia in the cat trunk with multifocally grouped, 2 to $8 \mathrm{~mm}$, pigmented, slightly raised, nonulcerated plaques (a). Detail of plaques arising from the alopecic and exfoliating skin (b): plaques have a rough surface and an irregular shape. Focal hyperplasia and dysplasia of the surface and follicular epidermis accompanied by mildly asynchronous keratinocyte maturation; note the loosely arranged and slightly fragmented collagen fibers in the dermis (c). Detail of (c): some keratinocytes have dark condensed nuclei and a clear cytoplasmic halo (koilocytes); others have a blue-grey cytoplasm (hematoxylin and eosin stain).

methylprednisolone acetate (Depo-Medrol Vet; Pfizer Italia S.r.l., Borgo San Michele, Italy) weekly and underwent full teeth extraction with the exception of the canines. Previously the cat had been administered undetermined courses of corticosteroids, without any detail about specific drug and dosages given by the owners. While general physical examination was unremarkable and mild stomatitis was seen at oral inspection, dermatological examination showed ill-defined alopecia on the trunk and flank with a very thin tissue paper skin, scaling, one wide laceration covered by necrotic material on the withers, and numerous small grouped slightly raised nonulcerated pigmented macules and plaques on the flank and posterior thorax (Figures 1(a) and 1(b)). Multiple skin scrapings and adhesive tape preparations showed no evidence of Demodex spp. mites, fungal culture was negative, and microscopic examination of hair shafts showed no abnormalities. A complete blood cell count and serum chemistry revealed mild leukocytosis (WBC $25,54 \mathrm{~K} / \mu \mathrm{L}$, reference values 5,50-19,50) with neutrophilia $(18,77 \mathrm{~K} / \mu \mathrm{L}$, reference values $2,50-12,50)$ and increase of mononucleated cells $(3,71 \mathrm{~K} / \mu \mathrm{L}$, reference values $0,15-1,70)$, hyperglycemia $(385 \mathrm{mg} / \mathrm{dL}$, reference values $71-159)$, and increased fructosamine levels $(396 \mu \mathrm{mol} / \mathrm{L}$, reference values 228-356). The cat was FeLV negative and FIV positive. $\mathrm{CBC}$ values, in particular persistent hyperglycemia, together with history of corticosteroid administration suggested iatrogenic hyperadrenocorticism. Liver lipidosis was considered unlikely for unchanged hepatic enzymes and for the good general condition of the cat. Clinical presentation indicated skin fragility and VP/BISC as main differential. Punch biopsies were taken from raised lesions, fixed in $10 \%$ buffered formalin, and routinely processed for histopathology. Histologically multiple sharply demarcated plaques of full-thickness epidermal and follicular dysplasia were seen, and groups of in situ proliferating keratinocytes showed loss of nuclear polarity (Figures $1(\mathrm{c})$ and $1(\mathrm{~d})$ ). A variable number of keratinocytes had cytoplasm enlarged by blue-greyish fibrillar material; some others had dark shrunken nucleus and clear cytoplasm (koilocytes). No invasion of the basal membrane into the dermis was detected. Epidermis adjacent to the neoplastic lesion was thin and keratotic, with collagen fibers of the underlying dermis markedly thin and loosely arranged. Collectively these features were indicative of SFS and concomitant multifocal VPs; despite the small dimension of the plaques, the possibility of progression to an early BISC was also indicated in the diagnostic report due to the follicular involvement and the dysplastic appearance of keratinocytes [8]. DNA was extracted from a fresh punch biopsy with the "DNeasy Blood and Tissue Kit" (Qiagen, Milan, Italy) according to vendor recommendations. To investigate the presence 


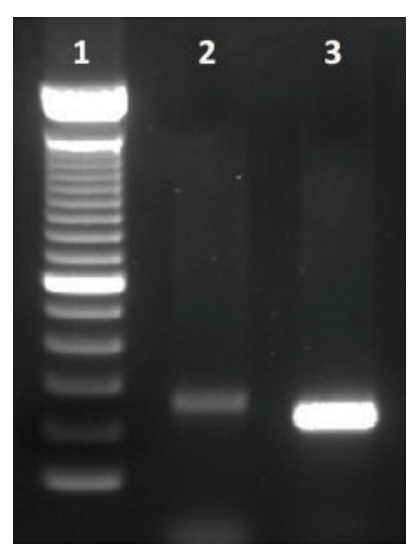

(a)

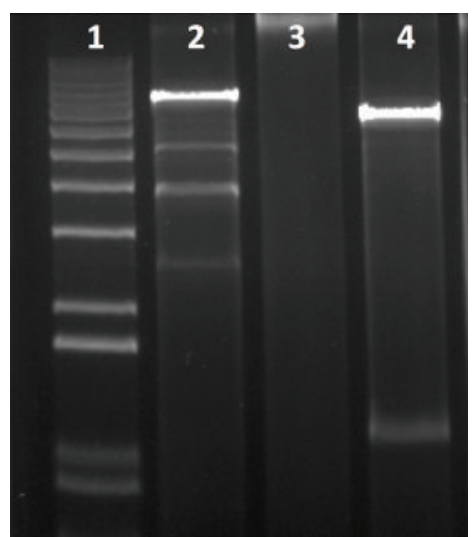

(b)

\begin{tabular}{lccr}
\hline & XbaI & EcoRI & \multicolumn{1}{c}{ SacI } \\
\hline FcaPV1 & - & $1(8300)$ & $2(1624+6676)$ \\
FcaPV2 & $2(2585+5314)$ & $3(1209+1778+4912)$ & $2(1531+6368)$ \\
FcaPV3 & $1(7583)$ & - & $2(6763+820)$ \\
FcaPV4 & $1(7616)$ & $1(7616)$ & $2(4881+2735)$ \\
Test sample & $1(8000-9000)$ & - & $2(7 / 8 \mathrm{~kb}+1200)$ \\
\hline
\end{tabular}

(c)

FIgure 2: Molecular detection and typing of feline papillomavirus. In (a) PCR results demonstrating the presence of at least two PV types are shown (1, Life Technologies 100 bp DNA Ladder; 2, 3, positivity to primers FPV12F/R and FPV34F/R, resp.). Results obtained with rolling circle amplification (RCA) (b) and restriction enzyme digestions of the RCA products (c) indicating the presence of FcaPV3 in the lesion.

of feline PVs 2 sets of primers were used. Primers FPV12F/R $\left(5^{\prime}\right.$-TGCAAATAYCCTGACTACAT- $3^{\prime} / 5^{\prime}$-CTATTRAAYAATTGRGCCTC- $3^{\prime}$ ) were designed to amplify $250 \mathrm{bp}$ of the L1 of FcaPV1 and FcaPV2. Similarly primers FPV34F/R ( $5^{\prime}$ - AGGACACTGAAAATCCCAAC- $3^{\prime} / 5^{\prime}$-TCACACATTTGACCATCCTC) targeted $230 \mathrm{bp}$ of the L1 of FcaPV3 and FcaPV4. PCR profiles were set according to vendor recommendation for Taq polymerase (Qiagen, Milan, Italy). Notably, PCR was positive for both primer's pairs, indicating the presence of at least 2 distinct papillomavirus types (Figure 2(a)). Amplicons FPV12F/R and FPV34F/R were successfully cloned into pCR4-TOPO (Invitrogen, Milan, Italy). Three clones per amplicon were automatically sequenced. Upon submission to standard nucleotide blast, sequences obtained from amplicon FPV12F/R (GeneBank accession number KP868617) revealed $100 \%$ of nucleotide identity with the corresponding L1 region of FcaPV2 reference strain (EU796884) and sequences derived from amplicon FPV34F/R (GeneBank accession number KP868618) shared 99\% of identity with FePV3 reference strain (JX972168). FcaPV2 and FcaPV3 viral loads were investigated by absolute quantification of DNA viral copies in the sample. Nucleic acid quantification was obtained by Real-Time PCR, performed with Rotor gene 3000 system (Corbett Research, Australia). With this aim, primers QFePV2F/R (5'-GCGGAGGGAGCAACAATACACT- $3^{\prime} / 5^{\prime}$-GGCCGCCTTCAAAACCAA- $\left.3^{\prime}\right)$ and QFePV3F/R ( $5^{\prime}$-GCACAGGGGAGCATTGG$3^{\prime} / 5^{\prime}$-GGTGGACAGTCCCCCTTTTG- $3^{\prime}$ ) were designed to amplify smaller fragments of the Felis catus papillomaviruses 2
(91 bp) and 3 ( $85 \mathrm{bp}$ ) L1, respectively. Absolute quantification of the viral DNA copies was obtained by plotting 10 -fold dilutions (from $1.2 \times 10^{1}$ to $1.2 \times 10^{4}$ copies $/ \mu \mathrm{L}$ ) of 2 distinct pUC19 plasmids containing the cloned fragments. Based on Real-Time PCR, 4555 copies/microliter of FcaPV2 and 8655 copies/microliter of FcaPV3 were present in the sample. Rolling circle amplification and restriction enzyme digestions resulted in the detection of a patter compatible with FcaPV3 (Figure 2(b)).

Based on clinical and histopathologic features, wearing of a bodysuit was recommended to prevent further skin tearing from itching. Therapy was initiated with doxycycline of $10 \mathrm{mg} / \mathrm{kg}$ once daily for 2 months; this drug has been chosen for its dual antimicrobial and immunomodulatory action. No further treatment was administered for the VPs. The followup in the first three months showed improvement in skin lesions: ulcers progressively healed, hair started to regrow, VPs were still present but again no therapy was underwent, and mild stomatitis was present at oral examination. After 18 months from initial diagnosis, on demand from the referring veterinarian, the owner was allowed to revisit the cat and the raised pigmented multiple plaque as well as skin fragility had resolved while mild stomatitis was still present.

Since early reports of feline skin hyperplastic lesions related to papillomavirus $[1,2]$ numerous other cases and series of cases have been published so far, in which PVs are considered to have a causative role in different feline skin diseases $[9,10]$. These include feline fibropapilloma or sarcoid 
[11] and a wide spectrum of proliferative skin diseases ranging from nonneoplastic VPs to preneoplastic BISC and invasive SSC [12]. Viral plaques, BISC, and SCC may coexist in the same skin lesion or the first may progressively evolve to the others in a long period of time. In our case the clinical and histopathological resemblance between VP and BISC lead to the risk of overdiagnosing a preneoplastic lesion (BISC); however, regression of VP after discontinuation of glucocorticoid and general condition improvement is in line with a nonneoplastic nature of the plaques. Some histological features of VP are shared with BISC and a clear separation between the two entities may be difficult. SFS is an uncommon acquired disease of cats that has been reported in association with several concurrent diseases, most commonly spontaneous or iatrogenic hyperadrenocorticism [12]. In the present case, skin fragility was noted at the time of presentation together with multiple raised plaques, and both lesions might have been induced by the previous glucocorticoid therapy; moreover, the cat was FIV positive. VPs have already been documented in cats with underlying FIV or glucocorticoid immunosuppression $[1,2,5]$. Cat pruritus was diffuse when the cat was found and it was not possible to ascertain whether VPs on the flank and thorax might have developed by inoculation of virus in self-induced skin abrasions. The regression of skin lesions in the case reported suggests that glucocorticoid therapy and FIV status might have predisposed to VP.

Four PV types have been so far described in cats $[6,9$, 13-15]. While association of FePV1 and FePV4 to disease remains to be clarified, an increasing number of reports indicate a correlation between the presence of FePV2 and FePV3 in VPs, BISCs, and SCCs $[5,13,16]$. FdPV-2 has been found in a high proportion of VPs and it has long been considered to be etiologically involved in the development of these skin lesions $[4,5]$; however, more recently, Munday and Witham [17] have amplified FcaPV2 from 50\% of swabs taken from the skin of clinically normal domestic cats, also suggesting a subclinical infection for this type of PV. A third PV, Feline Catus-PV3 (FcaPV3), has been also detected from a feline VP and BISC [4, 10]. Finally multiple PVs have been detected once in a single sample from a cat $[5]$.

\section{Conclusion}

This paper reports a case of VP in which, by combining PCR test with sequencing and Real-Time PCR, coinfection with $2 \mathrm{PV}$ types was recorded and their concentration was determined. Whether the role of the two viruses cannot be established in this case, FcaPV2 and FcaPV3 are present at comparable concentrations; it remains undetermined how each of the viruses might have contributed to the onset of lesions following immunosuppression. We hypothesize that one of the 2 viruses might have provided a more permissive environment for the other. Finally, spontaneous remission of skin lesions might have been induced by FIV status change over time due to glucocorticoid withdraw and by glucocorticoids withdraw itself.

\section{Conflict of Interests}

The authors declare that there is no conflict of interests regarding the publication of this paper.

\section{References}

[1] H. F. Egberink, A. Berrocal, H. A. D. Bax, T. S. G. A. M. van den Ingh, J. H. Walter, and M. C. Horzinek, "Papillomavirus associated skin lesions in a cat seropositive for feline immunodeficiency virus," Veterinary Microbiology, vol. 31, no. 2-3, pp. 117-125, 1992.

[2] H. C. Carney, J. J. England, E. C. Hodgin, H. E. Whiteley, D. L. Adkison, and J. P. Sundberg, "Papillomavirus infection of aged Persian cats," Journal of Veterinary Diagnostic Investigation, vol. 2, no. 4, pp. 294-299, 1990.

[3] J. L. Carpenter, J. W. Reider, J. Alroy, and G. M. Schmidt, "Cutaneous xanthogranuloma and viral papilloma on an eyelid of a cat," Veterinary Dermatology, vol. 3, no. 4-5, pp. 187-190, 1992.

[4] J. S. Munday and J. Peters-Kennedy, "Consistent detection of Felis domesticus papillomavirus 2 DNA sequences within feline viral plaques," Journal of Veterinary Diagnostic Investigation, vol. 22, no. 6, pp. 946-949, 2010.

[5] J. S. Munday, K. A. Willis, M. Kiupel, F. I. Hill, and M. Dunowska, "Amplification of three different papillomaviral DNA sequences from a cat with viral plaques," Veterinary Dermatology, vol. 19, no. 6, pp. 400-404, 2008.

[6] J. S. Munday, M. Kiupel, A. F. French, L. Howe, and R. A. Squires, "Detection of papillomaviral sequences in feline Bowenoid in situ carcinoma using consensus primers," Veterinary Dermatology, vol. 18, no. 4, pp. 241-245, 2007.

[7] H. Egberink, E. Thiry, K. Mostl et al., "Feline viral papillomatosis. ABC guidelines on prevention and management," Journal of Feline Medicine and Surgery, vol. 15, no. 7, pp. 560-562, 2013.

[8] T. L. Gross, P. J. Ihrke, and E. J. Walder, Skin Diseases of the Dog and Cat. Clinical and Histopathologic Diagnosis, Blackwell Science, Oxford, UK, 2nd edition, 2005.

[9] J. P. Sundberg, M. Van Ranst, R. Montali et al., "Feline papillomas and papillomaviruses," Veterinary Pathology, vol. 37, no. 1, pp. 1-10, 2000.

[10] J. S. Munday, M. Dunowska, S. F. Hills, and R. E. Laurie, "Genomic characterization of Felis catus papillomavirus-3: a novel papillomavirus detected in a feline Bowenoid in situ carcinoma," Veterinary Microbiology, vol. 165, no. 3-4, pp. 319325, 2013.

[11] F. Y. Schulman, A. E. Krafft, and T. Janczewski, "Feline cutaneous fibropapillomas: clinicopathologic findings and association with papillomavirus infection," Veterinary Pathology, vol. 38, no. 3, pp. 291-296, 2001.

[12] W. H. Miller, C. E. Griffin, and K. Campbell, "Viral, rickettsial and protozoal skin diseases," in Muller and Kirk's Small Animal Dermatology, pp. 350-351, Elsevier Mosby, St. Louis, Mo, USA, 7th edition, 2013.

[13] R. Tachezy, G. Duson, A. Rector, A. B. Jenson, J. P. Sundberg, and M. Van Ranst, "Cloning and genomic characterization of Felis domesticus papillomavirus type 1," Virology, vol. 301, no. 2, pp. 313-321, 2002.

[14] M. Dunowska, J. S. Munday, R. E. Laurie, and S. F. K. Hills, "Genomic characterisation of Felis catus papillomavirus 4, a novel papillomavirus detected in the oral cavity of a domestic cat," Virus Genes, vol. 48, no. 1, pp. 111-119, 2014. 
[15] M. Terai and R. D. Burk, "Felis domesticus papillomavirus, isolated from a skin lesion, is related to caine oral papillomavirus and contains a $1.3 \mathrm{~kb}$ non-coding region between the E2 and L2 open reading frames," Journal of General Virology, vol. 83, no. 9, pp. 2303-2307, 2002.

[16] J. S. Munday, "Papillomaviruses in felids," Veterinary Journal, vol. 199, no. 3, pp. 340-347, 2014.

[17] J. S. Munday and A. I. Witham, "Frequent detection of papillomavirus DNA in clinically normal skin of cats infected and noninfected with feline immunodeficiency virus," Veterinary Dermatology, vol. 21, no. 3, pp. 307-310, 2010. 



Submit your manuscripts at http://www.hindawi.com
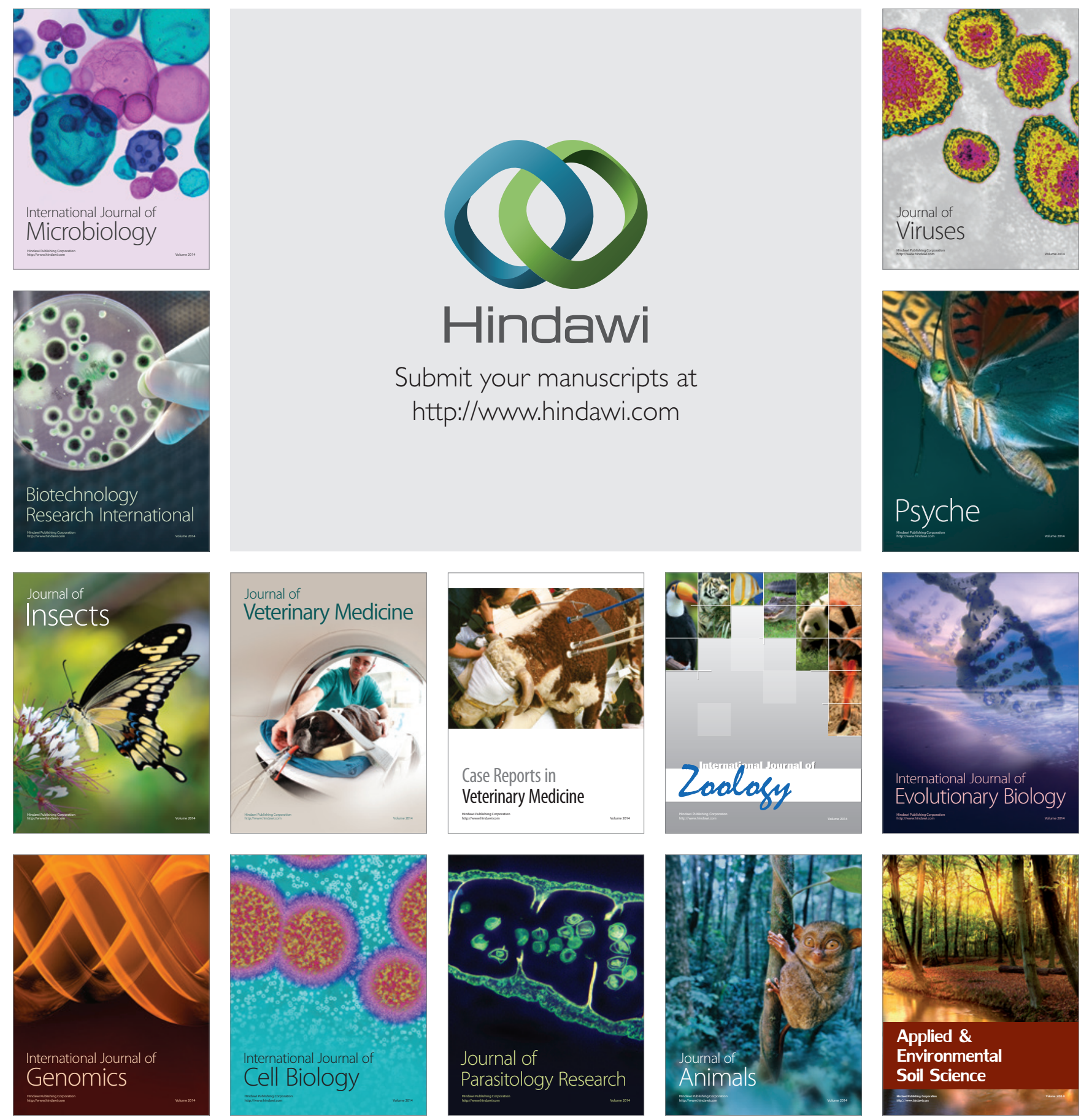